EPJ Web of Conferences 116, 03004 (2016)

DOI: $10.1051 /$ epjconf/201611603004

(C) Owned by the authors, published by EDP Sciences, 2016

\title{
Acoustic neutrino detection investigations within ANTARES and prospects for KM3NeT
}

\author{
Robert Lahmann ${ }^{\mathrm{a}}$ \\ Friedrich-Alexander-Universität Erlangen-Nürnberg, Erlangen Centre for Astroparticle Physics \\ (ECAP), Erwin-Rommel-Straße 1, 91058 Erlangen, Germany
}

\begin{abstract}
The acoustic neutrino detection technique is a promising approach for future large-scale detectors with the aim of measuring the small expected flux of cosmogenic neutrinos at energies exceeding $1 \mathrm{EeV}$. It suggests itself to investigate this technique in the context of underwater Cherenkov neutrino telescopes, in particular KM3NeT, because acoustic sensors are present by design to allow for the calibration of the positions of the optical sensors. For the future, the KM3NeT detector in the Mediterranean Sea will provide an ideal infrastructure for a dedicated array of acoustic sensors. In this presentation results from the acoustic array AMADEUS of the ANTARES detector will be discussed with respect to the potential and implications for acoustic neutrino detection with $\mathrm{KM} 3 \mathrm{NeT}$ and beyond.
\end{abstract}

\section{Introduction: Acoustic neutrino detection}

Measuring acoustic pressure pulses in huge underwater acoustic arrays is a promising approach for the detection of ultra-high-energy (UHE, $E_{v} \gtrsim 10^{9} \mathrm{GeV}$ ) neutrinos. These are expected to be produced in interactions of cosmic rays with the cosmic microwave background [1]. The pressure signals are produced by the particle showers that evolve when neutrinos interact with nuclei in water. The resulting energy deposition of the hadronic cascade within a cylindrical volume of a few centimetres in radius and several metres in length leads to a local heating of the medium. According to the thermo-acoustic model $[2,3]$, the accelerated expansion of the heated volume forms a pressure pulse of bipolar shape which propagates in the surrounding medium. Coherent superposition of the elementary sound waves, produced over the volume of the energy deposition, leads to a propagation within a flat disk-like volume (often referred to as pancake) in the direction perpendicular to the axis of the particle shower. After propagating several hundreds of metres in sea water, the pulse has a characteristic frequency spectrum that is expected to peak around $10 \mathrm{kHz}$ [4-6]. At these frequencies, the attenuation length of sound in sea water is about one to two orders of magnitude larger than that of Cherenkov light.

In this article, acoustic neutrino detection activities with a present (AMADEUS) and future (KM3NeT) experiment will be described and it will be pointed out how the experience with the former can be used for measurements with the latter.

\footnotetext{
a e-mail: robert.lahmann@fau.de
}

This is an Open Access article distributed under the terms of the Creative Commons Attribution License 4.0, which permits unrestricted use, distribution, and reproduction in any medium, provided the original work is properly cited. 


\section{Lessons learned from the AMADEUS project}

The AMADEUS project [7] was conceived to perform a feasibility study for a potential future largescale acoustic neutrino detector in the Mediterranean Sea. For this purpose, a dedicated array of acoustic sensors was integrated into the ANTARES neutrino telescope [8]. The detector is located in the Mediterranean Sea at the geographic position of $42^{\circ} 48^{\prime} \mathrm{N}, 6^{\circ} 10^{\prime} \mathrm{E}$ at a water depth of about $2500 \mathrm{~m}$, roughly $40 \mathrm{~km}$ south of the town of Toulon at the French coast. ANTARES comprises 12 vertical structures, the detection lines. Each detection line holds up to 25 storeys that are arranged at equal distances of $14.5 \mathrm{~m}$ along the line. A standard storey holds three optical modules, each one consisting of a photomultiplier tube inside a water-tight pressure-resistant glass sphere. A 13th line, the Instrumentation Line (IL), is equipped with instruments for monitoring the environment.

Within the AMADEUS system, acoustic sensing is integrated in the form of acoustic storeys with six acoustic sensors per storey, arranged at distances of roughly $1 \mathrm{~m}$ from each other. The AMADEUS system comprises a total of six acoustic storeys: three on the IL and three on the 12th detection line (Line 12). In April 2013, the IL was re-deployed at a new position at $150 \mathrm{~m}$ distance from Line 12. Until then, the distance between the two lines was $220 \mathrm{~m}$. The vertical distances between the two topmost acoustic storeys on the IL is increased from the standard of $14.5 \mathrm{~m}$ to about $110 \mathrm{~m}$.

To assess the feasibility of acoustic neutrino detection in a natural body of water, ambient noise and transient background at the site of the installation have to be investigated. The ambient noise is broadband and is mainly caused by agitation of the sea surface [9], i.e. by rain, wind, breaking waves, spray, and cavitations. It is predominantly the ambient background that determines the energy threshold for neutrino detection. Transient noise signals have short duration and an amplitude exceeding the ambient noise level. When of bipolar shape, these signals can mimic pulses from neutrino interactions. Sources of such signals can be anthropogenic, e.g. shipping traffic, or marine fauna. In particular dolphins emit short signals with a spectrum similar to that of acoustic emissions from neutrino interactions.

Two main lessons have been learned from the operation of the AMADEUS system. First, the ambient background is low and stable which is favourable for the operation of an acoustic neutrino detector. Second, the transient background has a high level. It is essential to reduce this background completely in order to measure the low expected rate of cosmogenic neutrinos.

A strong background reduction is already achieved with methods developed with AMADEUS [10]. Machine learning algorithms are used to identify bipolar pulses. Furthermore, events that are spatially and temporally clustered are discarded, as neutrinos are unlikely to produce several signals from one position within a short period of time. For further reduction it is essential to use the pancake shape of the signal as a selection criterion. This is not possible with AMADEUS due to its small size; such investigations are a main focus for the KM3NeT detector.

\section{Future activities in KM3NeT}

$\mathrm{KM} 3 \mathrm{NeT}$ is the next generation Cherenkov neutrino detector currently under construction in the Mediterranean Sea [11]. After its first phase of the construction, namely KM3NeT Phase 1, the detector will consists of 24 detection units (DUs) deployed off-shore Capo Passero in Italy (KM3NeT-IT), and 7 more DUs, deployed off-shore Toulon in France (KM3NeT-FR). Each DU holds a total of 18 optical modules [12], distributed equidistantly along flexible strings which for the KM3NeT-IT site have a length of about $700 \mathrm{~m}$. Each optical module contains 31 three-inch-photomultiplier tubes (PMTs) together with their readout electronics within a pressure-resistant glass spheres. When fully implemented, KM3NeT will eventually consist of several hundreds of DUs at multiple sites.

In order to determine the relative positions of the optical modules with a precision of not worse than $20 \mathrm{~cm}$, the detector will be equipped with an acoustic positioning system. The system employs acoustic 

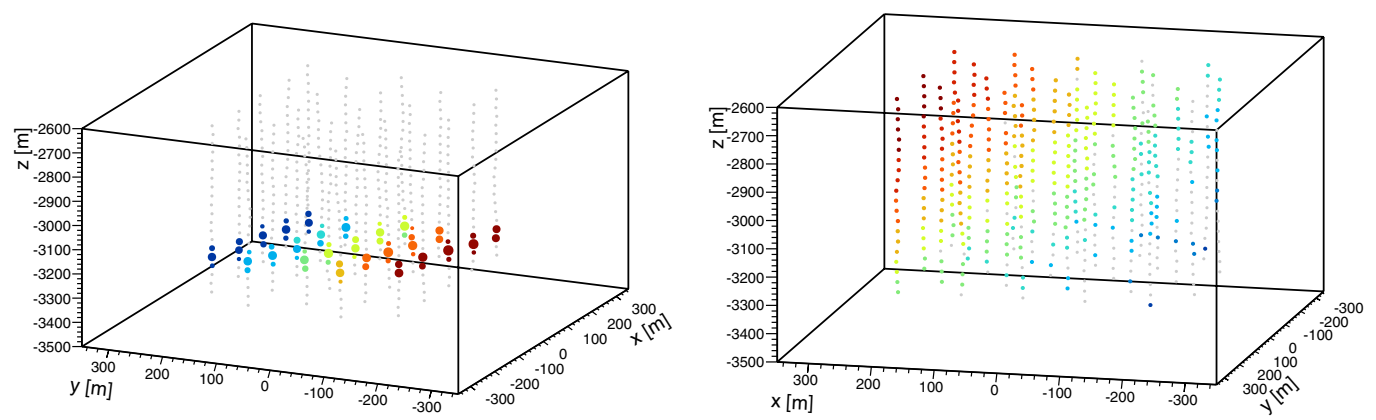

Figure 1. The signature of a neutrino event (left) and a spherical event originating from the surface of the sea, simulated in a KM3NeT-like acoustic detector with 25 lines spaced $90 \mathrm{~m}$ apart. Each dot represents an optical module with an integrated acoustic sensor, while the color indicates the arrival time of the acoustic signal (red: early, blue: late, grey: not triggered). The size of the dot represents the signal amplitude. The neutrino interacted at a distance of $1.8 \mathrm{~km}$ from the detector center with an energy of $10^{21} \mathrm{eV}$.

transceivers on the sea floor and acoustic receivers (hydrophones) in each storey and at the bases of the DUs. By performing multiple time-delay measurements and using these to triangulate the positions of the individual hydrophones, the hydrophone positions can be reconstructed relative to the positions of the emitters.

The design of the KM3NeT positioning system is based on experience with the systems developed for ANTARES and the former NEMO experiment, see [13] and references therein. Two types of acoustic sensors, both based on the piezo-electric effect, are used:

1. custom designed internal sensors. These are compact units of a piezo ceramic and a preamplifier, glued to the inside of the glass sphere of the optical module near its "South Pole";

2. external hydrophones, installed on the bases of the DUs.

Sampling is done at about $200 \mathrm{k}$ samples per second and all data is transmitted to shore. This way, algorithms for the position calibration running on an on-shore computer farm can be adapted to in-situ conditions that may affect the data quality. Furthermore, the data can be used for additional analyses, in particular acoustic detection of neutrinos, or marine science investigations.

The crucial questions for acoustic neutrino detection are: can neutrinos be classified backgroundfree? How low an energy threshold can be reached? And what volume needs to be instrumented to detect a predicted flux of cosmogenic neutrinos? These questions can be addressed by implementing the measurements for the ambient and transient noise from AMADEUS into simulations for acoustic neutrino detection with $\mathrm{KM} 3 \mathrm{NeT}$ and developing suitable filter and reconstruction algorithms.

To demonstrate the neutrino classification based on the shape of the acoustic emission pattern, a simulated neutrino signal and a background event for a detector configuration similar to that of KM3NeT-IT in Phase 1 are shown in Fig. 1 [14]. As can be seen, the size of the detector allows for a discrimination of the characteristic pancake shape against the spherical sound waves emitted from background events. First studies indicate that for an ambient noise and transient background as measured at the ANTARES site, the remaining background of neutrino-like events after suppression based on signal shape and clustering alone, as discussed in Sect. 2, can be reduced significantly when taking the emission pattern into account [14].

As a potential future extention to KM3NeT, the use of optical fibre-based hydrophones is under investigation [15]. To survey large volumes of water for acoustic signals from neutrino interactions, optical fibre-based hydrophones could potentially have several advantages over conventional hydrophones based on piezo ceramics. Investigations of adapting this technique to neutrino detection 
in combination with an under-water Cherenkov telescope are planned in the context of the KM3NeT experiment.

\section{Summary and conclusions}

First generation acoustic arrays such as AMADEUS have been used to investigate neutrino detection methods and provide input for simulations. Ambient noise could be shown to be low and stable while transient background is higher than expected and will require sophisticated methods of suppression. $\mathrm{KM} 3 \mathrm{NeT}$ provides an excellent framework for a second generation acoustic detection test setup - the detector is equipped with acoustic sensors for the purpose of position calibration which can also be used for neutrino detection investigations. Software tools developed for AMADEUS can be easily applied to KM3NeT. New concepts, in particular fibre-based hydrophones, can be used in the long run to further increase the instrumented volume of KM3NeT beyond the volume instrumented for optical detection of Cherenkov light.

The AMADEUS project is supported by the German federal ministry for education and research (BMBF) through grants 05A08WE1 and 05A11WE1.

\section{References}

[1] V.S. Berezinsky and G.T. Zatsepin, PL B 28, 423 (1969)

[2] G.A. Askariyan et al., Nucl. Inst. and Meth. 164, 267 (1979)

[3] J.G. Learned, Phys. Rev. D 19, 3293 (1979)

[4] S. Bevan et al. (ACoRNE Coll.), Astropart. Phys. 28, 366 (2007)

[5] S. Bevan et al. (ACoRNE Coll.), Nucl. Inst. and Meth. A 607, 398 (2009)

[6] V. Niess and V. Bertin, Astropart. Phys. 26, 243 (2006)

[7] J.A. Aguilar et al. (ANTARES Coll.), Nucl. Inst. and Meth. A 626-627, 128 (2011)

[8] M. Ageron et al. (ANTARES Coll.), Nucl. Inst. and Meth. A 656, 11 (2011)

[9] R.J. Urick, Ambient Noise in the Sea (Peninsula publishing, 1986), ISBN 0-932146-13-9

[10] M. Neff, Ph.D. thesis, Univ. Erlangen-Nürnberg (2013)

[11] The KM3NeT homepage, http://www.km3net.org/

[12] D. Vivolo, The KM3NeT Digital Optical Module, in Proceedings of VLVnT 2015, Rome, Italy, Sept. 14-16, 2015 (2016), to be published in EPJ Web of Conf.

[13] S. Viola et al., Acoustic positioning system for KM3NeT, in Proceedings of ICRC2015, The Hague, Netherlands, July 30-Aug. 6 (2016), to be published in: Proc. of Science

[14] D. Kießling et al., Simulation chain and signal classification for acoustic neutrino detection in seawater, in Proceedings of ARENA 2014, Annapolis, MD (USA), June 9-12 (2014), to be published in: AIP Conf. Proc.

[15] E.J. Buis et al., Fiber optic hydrophones for acoustic neutrino detection, in Proceedings of VLVnT 2015, Rome, Italy, Sept. 14-16, 2015 (2016), to be published in EPJ Web of Conf. 